\title{
Influences des Pressions Anthropiques sur les Lé- muriens d'Anantaka, dans la Partie Est du Plateau de Makira, Maroantsetra, Madagascar
}

David Rasolofoson', Gilbert Rakotondratsimba, Odon Rakotonirainy, Lucien M. A. Rakotozafy, Jonah H. Ratsimbazafy, Lydia Rabetafika, Rose M. Randrianarison

\section{RESUME}

L'équipe de Groupe d'Etude et de Recherche sur les Primates de Madagascar (GERP), pour contribuer dans la protection, a prospecté et suivi 12 sites dans la forêt de Makira dont Anantaka pendant les mois de septembre et octobre 2005 et 2006. A l'aide des lignes-inventaire, la densité relative de la population de lémuriens a été obtenue par des observations directes tandis que les pressions humaines ont été inventoriées par l'évaluation des surfaces d'incidence des activités humaines. Des activités anthropiques sur les lémuriens ont été identifiées. Les influences de chaque type de pression sur les espèces de Lémuriens (trois diurnes strictes, une cathémérale et dix nocturnes) répertoriées à travers 12 sites ont été analysées à partir du coefficient de corrélation $\mathrm{R}$ de Spearman entre chaque facteur menaçant par rapport à chaque effectif de lémurien. Ainsi, d'une part, on a déduit que le site d'Anantaka, avec ses 14 espèces lémuriennes, est le plus riche en espèces de lémuriens. Et d'autre part, on a constaté que plus le nombre de village est abondant et plus leur distance par rapport à la forêt est rapprochée et que les impacts de pression sur l'écosystème forestier augmente avec des activités relatives comme la chasse, les coupes illicites, les défrichements de la forêt primaire, les défrichements des forêts secondaires et la transformation des forêts en tavy ou en kijana (prairie) ainsi que les exploitations minières illicites. A part cette dégradation de la biodiversité, la décadence de la moralité à la fois sur les notions du civisme et le concept du fady (tabou) en l'encontre des Aye aye par exemple, se trouve sur une situation bouleversante, puisque les gens ne considèrent plus ni les valeurs culturelles pour la conservation de la forêt ni les lois régissant la gestion forestière et les chasses des animaux sauvages. Ces critères classifient le site d'Anantaka dans la cible focale de conservation du fait que la couverture forestière d'Anantaka présente encore 75 \% de forêt, $25 \%$ est transformée en savoka et en prairie. C'est ainsi que I'indispensablement d'une priorité en matière de conservation des lémuriens pour conserver la niche écologique exceptionnelle d'Anantaka. La conservation de Makira serait optimale avec I'intégration de la population riveraine du site d'Anantaka dans la gestion rationnelle de leurs ressources naturelles et avec I'intervention de l'éducation environnementale dans quelques localités dont les Communautés de base (COBA) et avec I'appui
Correspondance:

David Rasolofoson

34 Cité des Professeurs, Résidence Fort Duchesne, BP 779, Antananarivo 101, Madagascar

E-mail: gerp@moov.mg

technique émanant des associations dans I'élaboration d'un plan de développement touchant surtout I'agriculture et d'un projet qui assurera la surveillance et le contrôle du secteur par des missions de suivi-évaluation.

\section{INTRODUCTION}

Actuellement, les menaces influençant les écosystèmes forestiers de I'Est de Madagascar atteignent les forêts très reculées comme celles des forêts sur le plateau de Makira, à Maroantsetra, Madagascar. Ces forêts se situent dans la partie nord-est de Madagascar; elles constituent le plus grand bloc forestier contigu de la forêt dense humide sempervirente de la Grande Ile, avec ses 376,156 ha (Wildlife Conservation Society et Services des Eaux et Forêts 2004). Dans le but de mettre en place un plan de conservation des lémuriens dans ce bloc forestier, nous y avons exploré 12 différents sites (Figure 1). Parmi ces sites, Anantaka était prospecté et suivi pendant les mois de septembre et octobre 2005 et 2006. Anantaka est un écosystème forestier sur le flanc est du plateau de Makira ( $S$ : 15²5'52.7" EO: $49^{\circ} 27^{\prime} 16.0^{\prime \prime}$ ), dans le fokontany d'Andongona, commune rurale d'Antsirabe-Sahatany, à $45 \mathrm{~km}$ à I'ouest de Maroantsetra, région d'Analanjirofo, province de Toamasina. Pendant nos observations, des faits marquaient les enjeux environnementaux du site. Les paysans détruisent délibérément leurs milieux en défrichant illégalement la forêt pour pratiquer la culture sur brûlis et pour délimiter leur territoire. Cette délimitation est amorcée par l'installation de pièges à lémuriens, ensuite par le défrichement de la surface à exploiter. La présente étude consiste à déterminer l'influence des menaces anthropiques sur les lémuriens, dans l'optique de mettre en oeuvre les priorités en matière de conservation de ces animaux et d'intégrer la population riveraine du site à être responsable de la gestion rationnelle de leurs ressources naturelles.

\section{CARACTERISTIQUE DU SITE D'ANANTAKA}

Le site d'Anantaka est une forêt dense humide sempervirente évoluant sur un plateau migmatitique Archéen, associé à des fractures tectoniques et des émanations magmatiques du Précambrien, du Crétacé supérieur et du Tertio-Quaternaire de Madagascar (Besairie 1972). Sa position géographique est

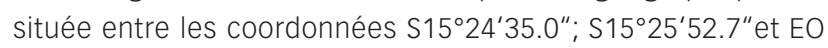


$49^{\circ} 27^{\prime} 12.2^{\prime \prime}$; EO 492 $27^{\prime} 16.0^{\prime \prime}$ (Figure 1); I'altitude varie entre 350 et 1,100 m. Le climat est de type tropical chaud et humide, avec une précipitation moyenne de 3,000 mm / an; les mois pluvieux vont de décembre en avril où la précipitation peut atteindre $2,500 \mathrm{~mm}$ et la saison sèche va du mois d'octobre à novembre où la précipitation varie de 80 à $120 \mathrm{~mm}$; la température oscille entre 15 et $35^{\circ} \mathrm{C}$ avec une moyenne de $24^{\circ} \mathrm{C}$; la forêt dans cette localité présente les caractéristiques de la zone éco-floristique orientale de basse à moyenne altitude, série à Anthostema et à Myristicaceae (Du Puy et Moat 1996; Humbert et Cours Darne 1965). Selon nos observations, les canopées forestières sont presque fermées et la plupart des hauteurs des arbres varient entre 20 et 30 m où leurs diamètres (dhp: diamètre hauteur de poitrine) sont souvent supérieurs à $30 \mathrm{~cm}$ tels que: Hazoambo ou Xylopia (Annonaceae), Notitrakoho ou (Aquafoliaceae), Vontro ou Dypsis, Rofilahy ou Ravenea (Arecaceae), Ramy ou Canarium (Burseraceae), Vongo ou Mammea, Vintanona ou Calophyllum, Azinina ou Symphonia (Clusiaceae), Fanjanabe ou Cyathea (Cyatheaceae), Hasimbe ou Dracaena (Dracaenaceae), Hazaomafana ou Diospyros (Ebenaceae), Vanana ou Sloaena (Elaeocarpaceae), Bakona ou Anthostema, Arina ou Bredelia, Paka ou Uapacca (Euphorbiaceae), Tanatanampotsy ou Tisonia (Flacourtiaceae), Tavolo ou Ocotea, Longotra ou Potameia (Lauraceae), Ambora ou Tambourissa (Monimiaceae), Ilondrara ou Brochoneura (Myristicaceae), Hompa ou Eugenia (Myrtaceae), Hetatra ou Podocarpus (Podocarpaceae), Hodopaso ou Prunus africanum (Rosaceae), Fatrena ou Breonia, Tifo ou Enterospermum, Taolanosy ou Gaertnera (Rubiaceae), Tamenapoza ou Sarcolaena (Sarcolaenaceae), Nanto ou Manilkara (Sapotaceae), Lombisy ou Rhopalocarpus (Sphaerosepalaceae).

\section{METHODOLOGIE}

RECENSEMENT PAR OBSERVATION DIRECTE DES

LEMURIENS Trois ligne-inventaires ont été utilisées pour le recensement des espèces de lémuriens diurnes, cathémérales et nocturnes, rencontrées le long d'un trajet de $2 \mathrm{~km}$ préalablement choisi. L'observateur se déplace à une vitesse de $0.7 \mathrm{~km}$ par heure et à chaque fois il rencontre un groupe d'animal, il s'arrête et note: I'heure de rencontre, la position géographique de l'endroit, le nombre d'individus rencontrés ainsi que leurs âges relatifs (adulte, jeune, petit) et leurs sexes (mâle ou femelle), la distance, perpendiculaire au trajet, entre I'animal et l'observateur, puis il reprend le déplacement à la même vitesse (Altmann 1974; Ganzhorn 1994). Les observations pendant la nuit se font avec I'utilisation de lumières artificielles atténuées (lampe frontale). Les tapetum lucidium sur la rétine des animaux nocturnes reflètent ces lumières, ce qui facilite leurs repérages. L'observateur utilise ensuite des lumières plus puissantes, pendant quelques minutes, pour déterminer l'espèce.

MODE DE CALCUL DE DENSITE Les densités relatives

de la population des lémuriens recensés pendant les périodes d'observations sont estimées à l'aide de la formule: $d=N / 2 \times W \times L$ (Avec d: densité estimée de la population, $\mathrm{N}$ : nombre d'animaux rencontrés, $\mathrm{W}$ : distance moyenne des animaux observés par rapport à la ligne-inventaire, L: Iongueur de la ligne-inventaire).

RECENSEMENT DES PRESSIONS HUMAINES POU

recenser les pressions humaines, nous avons procédé au comptage des traces d'activités humaines en suivant
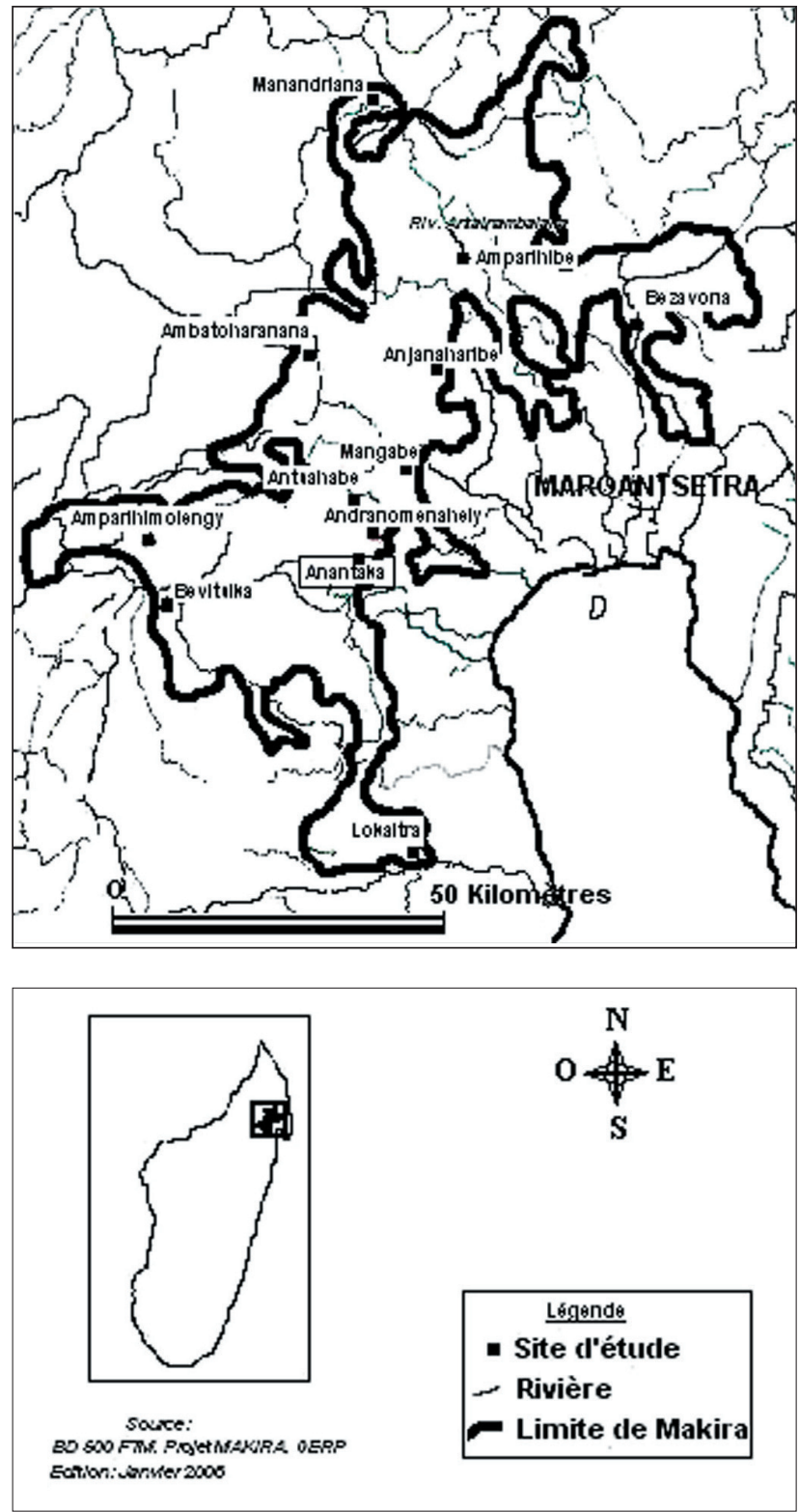

FIGURE 1. Localisation des sites d'études sur le site de conservation du plateau de Makira.

une ligne-inventaire et en quantifiant les produits exploités (comptage des arbres ou produits exploités dans la forêt) et puis en mesurant l'intensité des pressions anthropiques par l'évaluation des surfaces d'incidence des activités humaines à savoir les tavy rencontrés et les surfaces occupées.

ANALYSE DES DONNEES Dans cette étude, pour connaître

I'influence de chaque menace sur les lémuriens, nous avons calculé le coefficient de corrélation R de Spearman qui existe entre chaque facteur menaçant par rapport à chaque effectif de lémurien.

\section{RESULTATS ET INTERPRETATIONS}

LES LEMURIENS DANS LE SITE D'ANANTAKA 14 espèces

de lémuriens ont été inventoriées dans le site d'Anantaka. Parmi ces 14 espèces, trois sont diurnes strictes: Babakoto ou Indri indri, Varikandana ou Varecia variegata subcincta et Bokombolo ou Hapalemur griseus; une cathémérale: Varikosa ou Eulemur fulvus albifrons et dix nocturnes: Ampongy ou Avahi laniger, Tsitsihy ou Lepilemur seali, Hataka ou Chei- 
TABLEAU 2. Tableau des pressions recensées au niveau des différents sites dans le bloc forestier de Makira

\begin{tabular}{|c|c|c|c|c|c|c|}
\hline & \multicolumn{6}{|c|}{ PRINCIPAUX FACTEURS ANTHROPIQUES } \\
\hline & $\begin{array}{l}\text { Nombre de } \\
\text { villages interactifs }\end{array}$ & $\begin{array}{l}\text { Distance moyenne } \\
\text { entre un village et } \\
\text { le site forestier }(\mathrm{km})\end{array}$ & $\begin{array}{l}\text { Piège } \\
\text { (nombre / km²) }\end{array}$ & $\begin{array}{l}\text { Coupe illicite } \\
\text { (nombre de bois } \\
\text { de dhp }>18 \mathrm{~cm} \\
\text { coupés/ha) }\end{array}$ & $\begin{array}{l}\text { Surface sous forme } \\
\text { de tavy ou prairie } \\
\text { (kijana) dans le site (ha) }\end{array}$ & $\begin{array}{l}\text { Exploitation minière } \\
\text { (nombre de trous / ha) }\end{array}$ \\
\hline \multicolumn{7}{|l|}{ SITES } \\
\hline Manandriana & 5 & 2 & 0 & 186 & 75 & 600 \\
\hline Amparihibe & 6 & 20.65 & 0 & 0 & 0 & 0 \\
\hline Bezavona & 5 & 5.64 & 0 & 94 & 350 & 0 \\
\hline Ambatoharanana & 2 & 13.26 & 0 & 0 & 0 & 0 \\
\hline Anjanaharibe & 6 & 4.1 & 0 & 0 & 450 & 0 \\
\hline Mangabe & 5 & 9.26 & 0 & 255 & 30 & 0 \\
\hline Antsahabe & 1 & 13.17 & 0 & 32 & 0 & 0 \\
\hline Andranomenahely & 5 & 9.38 & 0 & 0 & 0 & 0 \\
\hline Anantaka & 6 & 5.98 & 66.7 & 266 & 300 & 0 \\
\hline Amparihimolengy & 6 & 15.98 & 0 & 0 & 500 & 0 \\
\hline Bevitsika & 2 & 10.29 & 0 & 0 & 0 & 0 \\
\hline Lokaitra & 7 & 9.95 & 7.81 & 54 & 200 & 232 \\
\hline
\end{tabular}

rogaleus major, Tsitsiha ou C. ravus, Tsitsihy ou C. sibreei, Microcèbe de Mittermeier ou Microcebus mittermeieri, Microcebus sp (en cours de détermination), Tsidiala ou Allocebus trichotis, Tantana ou Phaner furcifer et Hay hay ou Daubentonia madagascariensis.

A part, le site d'Anantaka, nous avons exploré 11 autres sites dans le bloc forestier de Makira, du nord au sud: Manandriana, Amparihibe, Bezavona, Ambatoharanana-Anjiabe, Anjanaharibe, Mangabe, Antsahabe, Andranomenahely, Ambongabe Amparihimolengy, Maroankolany Bevitsika et Lokaitra. Le nombre d'espèces de lémuriens dans ces autres sites varie entre trois et 12. Le Tableau 1 suivant résume les nombres d'espèces recensées dans le bloc forestier de Makira, en fonction de leur rythme nycthéméral.

Les 12 sites visités au bloc forestier de Makira présentent 20 espèces de lémuriens; parmi les espèces recensées $60 \%$ sont nocturnes, $25 \%$ diurnes et $15 \%$ cathémérales. La comparaison des 12 sites répertoriés permet de déduire que le site d'Anantaka est le plus riche en espèces de lémuriens où on y a recensé au total 14 espèces, suivi de Mangabe, Antsahabe, Andranomenahely, Ambongabe-Amparihimolengy et de Lokaitra avec 12 espèces, puis Ambatoharanana-Anjiabe, Anjanaharibe et Maroankolany-Bevitsika avec dix espèces; ensuite Bezavona avec neuf espèces, Manandriana avec sept espèces et en dernier Amparihibe qui n'abrite que trois espèces. Par rapport à l'orientation des sites d'études, la partie centrale et la partie sud du bloc forestier présentent une homogénéité d'espèces lémuriennes par rapport à la partie nord. Le site d'Anantaka, avec ses 14 espèces de lémuriens, présente donc une richesse très élevée en diversité spécifique lémurienne par rapport aux 11 sites que nous avons étudiés dans le bloc forestier du plateau de Makira et par rapport aux autres sites déjà explorés dans les forêts de Makira (Raharivololona et al. 2003) ainsi qu'aux aires protégées environnantes comme Anjanaharibe Sud, Marojejy et Masoala (Schimd et Smolker 1998; Sterling et Rakotoarison 1998; Sterling et McFadden 2000).
LES PRESSIONS HUMAINES Plusieurs facteurs anthropiquesmenacent l'écosystème forestier dans le plateau de Makira. Le Tableau 2 ci-dessous indique les pressions relatives à chaque site.

Le Tableau 2 présente les valeurs de pressions anthropiques recensées par site dans le bloc forestier de Makira. Nous constatons que plus le nombre de villages est abondant et plus leur distance par rapport à la forêt est rapprochée, les impacts de pression sur l'écosystème forestier augmente. La chasse, les coupes illicites, les défrichements de la forêt primaire, les défrichements des forêts secondaires et la transformation des forêts en 'tavy' ou en prairie 'kijana' ainsi que les exploitations minières illicites peuvent influer l'équilibre écologique forestier. Dans le bloc forestier de Makira, les sites de Manandriana, d'Anantaka et de Lokaitra sont les plus exposés aux menaces anthropiques par rapport aux neuf autres sites mais la spécificité du site d'Anantaka oriente notre étude sur l'influence des menaces anthropiques sur les lémuriens.

RELATION ENTRE LES PRESSIONS ANTHROPIQUES ET

LES ESPECES DE LEMURIENS La chasse et le piégeage sont très courants à Anantaka pour satisfaire les besoins rationnels en protéine animale; les chasseurs pratiquent à la fois la chasse traditionnelle, en utilisant des pièges à lémuriens ('laly'), des frondes et parfois des fusils de chasse. Ces pratiques atteignent leurs paroxysmes aux environs des périodes de festivité, telles que la fête pascale, la fête nationale et les fêtes de la fin d'année. Elles sont également importantes pendant la période de labour de rizière pour valoriser les travaux effectués, sous forme d'entraide dans la société. Les résultats de nos enquêtes indiquent que les lémuriens les plus recherchés sont ceux de grande taille comme: Indri indri, Varecia variegata subcincta et Eulemur fulvus albifrons et si les pièges attrapent des Daubentonia madagascariensis, les chasseurs les ramènent et les mangent comme tous les proies. Après la chasse, au moins une personne ramène cinq à dix individus, d'une même ou de différentes espèces. Si la chasse est bonne, une partie est consacrée à la famille et les restes sont vendus aux clients 
Tableau 3. Influence des pièges et des coupes d'abres sur les lémuriens dans le site d'Anantaka

\begin{tabular}{|l|l|l|l|}
\hline ESPECES DE LEMURIENS & $\begin{array}{l}\text { DENSITE DE LEMURIENS } \\
\text { (INDIVIDU/KM }{ }^{2}\end{array}$ & $\begin{array}{l}\text { COEFFICIENT DE CORRELATION } \\
\text { AVEC LE PIEGE }\end{array}$ & -0.35 \\
\hline Indri indri & 13 & -0.61 & -0.77 \\
\hline Varecia variegata subcincta & 5 & 0 & -0.93 \\
\hline Hapalemur griseus griseus & 1 & -0.08 & 0 \\
\hline Eulemur fulvus albifrons & 57 & -0.01 & -0.46 \\
\hline Avahi laniger & 65 & -0.02 & -0.43 \\
\hline Lepilemur seali & 30 & -0.12 & -0.62 \\
\hline Cheirogaleus major & 42 & -0.32 & -0.54 \\
\hline Cheirogaleus ravus & 15 & -0.51 & -0.73 \\
\hline Cheirogaleus sibreei & 7 & -0.12 & -0.9 \\
\hline Microcebus mittermeieri & 40 & -0.63 & -0.56 \\
\hline Microcebus sp. & 6 & -0.32 & -0.9 \\
\hline Allocebus trichotis & 14 & -0.61 & -0.77 \\
\hline Phaner furcifer & 5 & -0.93 \\
\hline
\end{tabular}

habituels qui ont effectués leurs commandes au préalable. La pratique de chasse de lémuriens par l'installation des pièges sous formes de 'laly' et 'laly totoka' (système de piège où l'on essaie de cerner l'habitat d'un groupe de lémuriens en créant un passage forcé pour piéger un animal) menace directement ces animaux, car une telle installation défriche directement le domaine vital d'un groupe et qui provoque l'éclatement ou I'isolement d'un groupe. En effet, pour installer une piège, une surface de $815 \mathrm{~m}^{2}$ de forêt est détruite et 14 arbres de dhp $>18$ $\mathrm{cm}$ sont abattus. Le Tableau $3 \mathrm{ci}$-après rapporte les résultats des calculs de corrélations entre les pièges recensés au niveau du site d'Anantaka et la densité au $\mathrm{km}^{2}$ de chaque espèce de lémuriens rencontrée lors des suivis des ligne-inventaires.

$L^{\prime}$ analyse de coefficient de Spearman donne $R<0.5324$, $\alpha=0.05$ avec $N=12$ (nombre des sites), non significatif: $c^{\prime}$ est à dire, il n'y a pas de corrélation entre les facteurs humains et la densité relative des lémuriens. En outre, les coefficients de corrélation de chaque espèce de lémuriens avec le piège montrent une valeur négative telle que: plus le nombre de piège augmente, plus la densité de chaque espèce de lémuriens diminue, c'est-à-dire toutes les espèces de lémuriens à Anantaka sont influencées par les installations des pièges. Par rapport aux valeurs du coefficient de corrélation $\mathrm{R}$, les espèces de lémuriens suivantes: Varecia variegata subcincta, Cheirogaleus sibreei, Microcebus sp, Phaner furcifer et Daubentonia madagascariensis ont des valeurs supérieures à 0.5324. Ces espèces sont donc influencées par les pièges. Par contre, les autres espèces: Indri indri, Eulemur fulvus albifrons, Avahi laniger, Lepilemur seali, Cheirogaleus major, C. ravus, Microcebus mittermeieri et Allocebus trichotis ne sont pas influencées aux pièges, car la valeur de leurs coefficients de corrélation R est inférieure à 0.5324. Donc, chaque espèce de Lémuriens peut être sensible ou non aux pièges sauf I'Hapalemur griseus qui présente une valeur de R égale à zéro. Mais cette espèce est vulnérable car sa densité est faible. Par rapport aux coupes d'arbres, les coefficients de corrélation de chaque espèce de lémuriens montrent une valeur négative. La corrélation négative indique que: plus le nombre de coupe d'arbres augmente, plus la densité de chaque espèce de lémuriens diminue, c'est-à-dire que toutes les espèces de lémuriens à Anantaka sont influencées par les coupes d'arbres. Par rapport aux valeurs du coefficient de corrélation R; les espèces de lémuriens suivantes: Eulemur fulvus albifrons et Avahi laniger ont des valeurs inférieures à 0.5324 ; c'est-à-dire que ces deux espèces sont insensibles aux coupes d'arbres. Par contre, la valeur du coefficient de corrélation R est supérieure à 0.5324 pour les espèces suivantes: Indri indri, Varecia variegata subcincta, Lepilemur seali, Cheirogaleus major, C. ravus, C. sibreei, Microcebus mittermeieri, Microcebus sp., Allocebus trichotis; Phaner furcifer et Daubentonia madagascariensis, lesquelles sont de ce fait sensibles aux coupes d'arbres. Donc, chaque espèce de lémuriens peut être sensible ou non aux coupes d'arbres sauf Hapalemur griseus qui, ayant une valeur de R égale à zéro, est vulnérable à cause de sa faible densité.

Dans le site d'Anantaka, $36 \%$ des espèces de lémuriens sont sensibles aux pièges et $64 \%$ en sont insensibles et par rapport aux coupes d'arbres, $85 \%$ des espèces de lémuriens existantes sont sensibles et $15 \%$ insensibles. Donc, les facteurs anthropiques les plus menaçants d'Anantaka sont les coupes d'arbres et l'installation de pièges. Les destructions de I'habitat conduisent au déséquilibre de l'écosystème forestier, à la décadence de la biodiversité et à la dégradation des différentes niches écologiques. A part cette dégradation de la biodiversité, la décadence de la moralité à la fois sur les notions du civisme et le concept du tabou ('fady') se trouve sur une situation bouleversante, puisque les gens ne considèrent plus ni les valeurs culturelles pour la conservation de la forêt ni les lois régissant la gestion forestière et les chasses des animaux sauvages.

\section{RECOMMANDATION ET CONSERVATION}

PRIORITE EN MATIERE DE CONSERVATION DES

LEMURIENS SUR LES 90 espèces de lémuriens connues actuellement à Madagascar (Mittermeier et al. 2006), Anantaka, présente 14 espèces (15\%) regroupées dans les cinq familles de lémuriens actuelles. Avoir de tel nombre d'espèces lémuriennes dans un même site est rarissime. L'habitat d'Anantaka présente donc une niche écologique encore originelle conven- 
able aux différentes espèces de lémuriens adaptées aux forêts denses humides de basse à moyenne altitude de l'est de Madagascar. Ces critères classifient ce site d'Anantaka dans la cible focale de conservation. Comme les lémuriens sont des animaux arboricoles, le défrichement de la forêt pour la culture sur brûlis (culture de riz, cultures vivrières) ou pour l'extension des propriétés foncières ainsi que l'installation des pièges à lémuriens menace leurs existences. Trois paramètres priorisent la conservation des espèces, à savoir, la superficie de I'habitat à conserver, la structure et les conditions écologiques de l'habitat et la relation de I'habitat à conserver avec I'habitat périphérique (SAPM/UICN 2005). La couverture forestière d'Anantaka présente encore $75 \%$ de forêt, $25 \%$ est transformée en 'savoka' et en prairie; Anantaka est un habitat exceptionnel pour les diversités lémuriennes qui nécessitent un système de conservation dans l'immédiat.

INTEGRATION DE LA POPULATION RIVERAINE DU

SITE D'ANANTAKA À ÊTRE RESPONSABLE DE LA GESTION RATIONNELLE DE LEURS RESSOURCES NATURELLES En 2004, la politique de gestion forestière GELOSE (Gestion locale sécurisée pour gérer les forêts communautaires) est appliquée dans la zone. Le projet émane du WCS/Projet Makira et le service de Cantonnement de Maroantsetra. Alors, on a crée les communautés de base (COBA) à Anjiahely et à Antsirabe, villages aux alentours d'Andongona et d'Anantaka. L'impact de cette implantation semble immédiat car la pratique de la chasse, de culture sur brûlis et les défrichements illicites de forêt sont atténués pour ces villages car les communautés elles-mêmes gèrent leurs forêts. Par contre, cette initiative n'a pas d'impact dans le site d'Anantaka, car la chasse et le piégeage ainsi que le défrichement des forêts continuent toujours. Pendant nos observations en 2006, 20 pièges ont été repérés par comptage direct au niveau des ligne-inventaires du site d'Anantaka, dont la mise en place entraîne la destruction d'une espace forestière de 1,6257 $\mathrm{m}^{2}$, où environ 266 arbres de $\mathrm{dhp}>18 \mathrm{~cm}$ sont abattus. L'abondance des pièges accélère la destruction de la forêt et souvent les chasseurs n'arrivent plus à collecter les animaux piégés et les laissent pourrir sur place où ils deviennent des proies faciles pour les Cryptoprocta ferox, nous en étions des témoins oculaires dans le cas d'un Tsitsiha ou Cheirogaleus ravus (Figure 2).

L'intervention de l'éducation environnementale est indispensable dans le 'fokontany' d'Andongona et le village d'Anantaka pour sensibiliser les COBA et les populations sur I'importance et l'avantage des ressources forestières, pour qu'ils ne défrichent plus la forêt mais de restaurer plutôt les forêts détruites par les pratiques de cultures sur brûlis et I'installation des pièges à lémuriens. Les appuis techniques seront indispensables pour les associations à élaborer un plan de développement tenant compte de la nécessité de fournir à ses membres des services de qualité, de développer sa viabilité financière. L'application de politiques économiques relatives à satisfaire les besoins quotidiens de chaque membre de I'association est primordiale, c'est-à-dire faire un aménagement de terroir pour permettre les cultures vivrières et d'instaurer l'équilibre des circuits de production et de consommation dans un système économique adéquat. Ce système nécessite la création des conditions améliorant I'offre viable en produits et en services financiers, dans les zones rurales. Donc, ce système nécessite la création d’un projet qui assurera

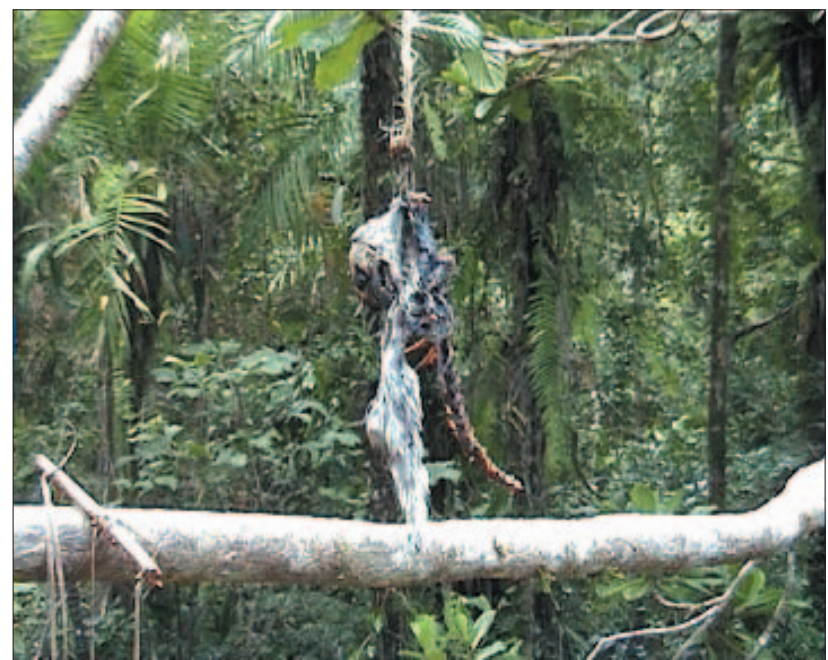

FIGURE 2. Cheirogaleus ravus oublié par le chasseur et dévoré par le Fosa ou Cryptoprocta ferox

la surveillance et le contrôle du secteur par des missions de suivi-évaluation et tenir des rencontres de concertation pour des éventuels ajustements adaptés et afin de mieux sécuriser les différents appuis financiers et techniques.

\section{CONCLUSION}

L'intensité des pressions humaines est très forte particulièrement dans le site d'Anantaka à cause des installations des pièges à lémuriens qui entraînent la fragmentation et la dégradation partielle de la forêt. Ces fragmentations amplifient la dégradation produite par la pratique de culture sur brûlis. Ces situations perturbent la biodiversité dans le site, en particulier celle des lémuriens, car les fragmentations de leurs habitats naturels diminuent les espaces de leurs domaines vitaux. Ces perturbations pourront entraîner par la suite la migration de l'espèce animale et même la disparition progressive des quatorze espèces existantes ainsi, Anantaka ne pourra plus être un site de conservation exceptionnelle pour les lémuriens. Le paradoxe de la pratique de culture sur brûlis comme dans toute la côte Est de Madagascar se fait sentir dans cette zone, car les besoins en rations alimentaires humaines riment avec la destruction des ressources naturelles à partir de laquelle le système de gestion et de conservation n'est plus respecté. Les influences des pressions anthropiques n'affectent pas seulement les lémuriens mais elles peuvent également avoir des conséquences sur la dégradation du biotope et sur le mode de vie de l'homme. En effet, une visualisation des appuis techniques sur l'agriculture et une activité génératrice de revenus sur les cultures vivrières pour satisfaire les besoins locaux sont indispensable dans l'immédiat pour nourrir les 2,300 habitants. Ensuite, il faut clarifier aux gens, l'importance des ressources naturelles, I'équilibre écologique et son impact dans la vie quotidienne, la nécessité de la gestion de ces ressources dans une vision durable, par le biais de l'éducation environnementale pour améliorer d'une manière stable leurs niveaux de vie.

\section{REMERCIEMENTS}

Nous adressons nos remerciements à la Fondation MacArthur qui a financé le présent projet et au projet Wildlife Conservation Society (WCS) Madagascar en particulier WCS / Projet Makira, Maroantsetra et à toutes ses équipes, dirigés par son Directeur 
National Monsieur Jean Jacques Jaozandry et le Conseiller Technique Principal Monsieur Christophe Holmes pour leurs aimables collaborations, ainsi qu'à toutes Autorités administratives (Direction de la Préservation de la Biodiversité du Ministère de I'Environnement et des Eaux et Forêts, Direction des Eaux et Forêts de Toamasina et ses collaborateurs, les Circonscriptions des Eaux et Forêts d'Antsohihy, de Fénérive Est et d'Antalaha, les services de cantonnements forestiers de Maroantsetra, d'Andapa, d'Antalaha, de Mandritsara et de Befandriana, les Chefs de District de Maroantsetra, de Mandritsara et de Befandriana, les Maires dans les différentes communes), les autorités locales (Chefs de fokontany, Tangalamena et Sojabe) de Maroantsetra, Mandritsara et de Befandriana, les Communautés de base aux environs de nos sites d'interventions pour leurs appuis en ressources humaines (Guides et Porteurs), en matériels et logistiques et en particulier à tout le Staff de MCD, les Reviewers pour leurs précieux commentaires prodigués sur cet article.

\section{REFERENCES}

Altmann, J. 1974. Observational study of behavior: Sampling methods. Behavior 49: 227-267.

Besairie H. 1972. Géologie de Madagascar, Service Géologique de Madagascar. Tananarive.

Commission Communication SAPM (Système des Aires Protégées à Madagascar). 2005. Adhésion des cibles à différent niveau à la mise en place du SAPM (non publié).

Du Puy, M. 1996. A refined classification of primary vegetation of Madagascar based on the underlying geology: using GIS to map its distribution and to cossets conservation status. W.R Laurence (éds.) Proceedings of the international symposium on the biogeography of Madagascar: 205-218 + 3 maps. Edition de I'ORSTOM. Paris.
Fonds d'Intervention pour le Développement (FID). 2003. Plan Communal de Développement de la commune rurale d'Antsirabe Sahatany (non publié).

Ganzhorn, J. U. 1994. Inventaires biologiques dans la forêt de Zombitse. Les Lémuriens. Goodman S. M., Langrand, O. (éds.), Recherche pour le développement, Série Sciences biologiques, CIDST, Antananarivo, Madagascar, Numéro Spécial, pp. 70-72.

Humbert, H., Cours- Darne, G. 1965. Description des types de végétation. H. Humbert et G. Cours Darne (éds.), Notice de la carte de Madagascar - travaux de la section scientifique et technique de I'Institut français de Pondichéry, 6:46-78.

Ministère de I'Environnement, des Eaux et Forêts, WCS, 2003. Site de Conservation de Makira: Rapport d'atelier de planification technique et stratégique, février 2003. (non publié).

Mittermeier, R.A., Konstant W.R., Hawkins, F., Louis E.E., Langrand, O., Ratsimbazafy, H.J., Rasoloarison, R., Ganzhorn, J.U., Rajaobelina, S., Tattersall, I., Meyers, D.M. 2006. Lemurs of Madagascar (Second edition), Conservation International Tropical Field Guide Series, Washington, D.C. 520 p.

Raharivololona, B.M., Ratsisetraina, R.I., et Day, S.R. 2003. Lémuriens des forêts humides du plateau de Makira, Maroantsetra, Madagascar. 2003. Lemur News, 8:18-19.

Schmid, J. and Smolker, R. 1998. Lemurs of the Reserve Spéciale d'Anjanaharibe-Sud, Madagascar; Fieldiana Zoology 90:227-240.

Sterling, E.J. and McFadden, K. 2000. Rapid census of lemur populations in Parc National de Marojejy, Madagascar. S.M. Goodman (eds.), A floral and Faunal Inventory of the Parc National de Marojejy, Madagascar: With Reference to elevational variation. Fieldiana Zoology, New series 97: 265-274

Sterling, E.J. and Rakotoarison, N. 1998. Rapid assessment of richness and density of primate species on the Masoala peninsula, eastern Madagascar. Folia Primatologica, 69:109-116.

Wildlife Conservation Society. 2004. Contexte environnemental du site de conservation de Makira (Rapport pour le WCS, non publié). 